\title{
Fine-tuning and cloning of a fiber-optic probe for in situ monitoring and evaluation of quality of olive oil products
}

\author{
D. Pérez-Marín, ${ }^{1, a}$ J.A. Adame-Siles, ${ }^{2, a}$ F. Sánchez-Müller, ${ }^{a}$ F. Maroto-Molina ${ }^{a}$ and A. Garrido-Varo ${ }^{a}$ \\ ${ }^{a}$ Agro-livestock Systems Engineering Department, Non-destructive Sensors Unit, University of Córdoba, Campus Rabanales, N-IV, km 396, Córdoba, \\ 14014, Spain. E-mail: ${ }^{1}$ dcperez@uco.es, ${ }^{2}$ g42adsij@uco.es
}

The control of conformity at reception of bulk olive oil before unloading is an essential step for packing plants to meet quality standards and ensure traceability. New approaches, different to sampling and analysis at-line, are needed to improve efficiency and decision-making processes. Near-infrared spectroscopy (NIRS) provides fast, cost-effective and in situ analytical measurements without sample preparation, which makes it an ideal technology towards this goal. The purpose of this work is to assess and optimize the spectral acquisition with a fiber-optic probe, especially designed to sample and analyze tank trucks. This probe features two fiber optic bundles, one of measurement and one of illumination. First of all, two olive oils samples, one of extra virgin and one of lampante category, were used to evaluate the noise and repeatability of the NIRS spectral signals of the target probe. A set of 20 spectra were recorded for each sample, and the sequence of measurements lampante-extra virgin was repeated three times per day for seven days spread over five weeks. The noise level was evaluated using a first derivative pre-treatment, and the optimum working range (after removing the noisy regions) was $1150-2149 \mathrm{~nm}$. The Root Mean Square (RMS) statistic and the MEAN values were calculated in each case, and the spectral repeatability results showed that the RMS (MEAN) values for lampante were higher than for extra virgin olive oil (12,630 and $2998 \mu \log (1 / R)$, respectively). Furthermore, a standardization procedure was performed to make a comparative study with a laboratory probe. Both probes were connected to a MATRIX-F Fourier transform-NIR instrument. A set of 42 samples of different nature were used for standardization purposes. Two matrices of standardization were developed, selecting 1 (STD1) and 10 (STD2) samples, and a validation set of 32 samples was used. The spectral adjustment was evaluated using the validation set by calculating the RMS(c) statistic. The RMS(c) values obtained in the master (laboratory probe, LP) versus satellite (in situ analysis probe, IAP) before standardization showed important differences that were significantly corrected by the best standardization matrix (STD1).

\section{Introduction}

Food safety and quality assurance are key pillars of EU legislation. In this context, EU food policy comprises an integrated approach, which involves the need to implement mechanisms and effective programs performing monitoring, surveillance and process control along the entire agri-food chain. ${ }^{1}$ As a consequence, competent bodies and agri-food system stakeholders are currently faced with a number of challenges when it comes to ensuring traceability, regulatory compliance and quality standards at all stages.

Furthermore, this framework is pushing manufacturers to continuously improving their processes and practices.
Nowadays, not only do they need to meet existing regulations and standards but also consumers' requirements, who want to be better informed and are increasingly demanding safer and higher quality products. The olive sector is part and parcel of the EU model of agriculture, having a significant social and economic impact, and needs to tackle a myriad of difficulties in this regard as well.

From the olive oil industry viewpoint, packing plants play a crucial intermediary role. They handle, store and process the olive oils produced at mills from different geographical locations, and their final output is intended for marketing for

\section{Correspondence}

D. Pérez-Marín, J.A. Adame-Siles (dcperez@uco.es, g42adsij@uco.es) doi: 10.1255/nir2017.115

Citation: D. Pérez-Marín, J.A. Adame-Siles, F. Sánchez-Müller, F. MarotoMolina and A. Garrido-Varo, "Fine-tuning and cloning of a fiber-optic probe for in situ monitoring and evaluation of quality of olive oil products", in Proc. $18^{\text {th }}$ Int. Conf. Near Infrared Spectrosc., Ed by S.B. Engelsen, K.M. Sørensen and F. van den Berg. IM Publications Open, Chichester, pp. 115-121 (2019). https:// doi.org/10.1255/nir2017.115

\section{(c) 2019 The Authors}

This licence permits you to use, share, copy and redistribute the paper in any medium or any format provided that a full citation to the original paper is given, the use is not for commercial purposes and the paper is not changed in any way.

ISBN: 978-1-906715-27-4 
direct consumption after labelling and packaging. There are many critical control points across the stages of the production process at a packing plant (storage, blending, filtering, packing etc.). ${ }^{2}$ However, the importance of the inspections at the reception level should be highlighted. One the one hand, they make it possible to detect any hazards, either due to the presence of foreign matter, contaminants or residues, before the product enters the process. Besides food risk management, control of raw materials at this point is also vital for the purpose of standards compliance and traceability. Sampling and physicochemical and organoleptic analysis of olive oil delivery vehicles are of great value as far as quality assurance is concerned. They are essential for a successful and reliable management system, in which the product ought to be correctly assigned to the most appropriate storage tank or lot according to its quality. Last but not least, compliance checks at delivery enable the industry to determine and guarantee that the olive oil corresponds to the category for which the contract was negotiated. Thus, inspection of delivery vehicles is key in terms of product acceptance to detect possible frauds and to validate whether suppliers and transportation are complying with the certification. If that is not the case, otherwise the industry might reject the load before compromising safety/quality in subsequent steps.

Bulk raw materials sampling and analysis are not without difficult issues to address. Logistical, operational and methodological problems arise when evaluating large volumes of sample. Most existing protocols aimed at assessing quality of olive oil tanker trucks require the mechanical withdrawal of samples. ${ }^{3}$ Then, these samples are usually sent pneumatically to the laboratory for physico-chemical testing, where classical techniques are frequently used to characterize the lot. Despite traditional methods of sampling and analysis are widespread, they have some critical drawbacks which include: low samples throughput, high costs of implementation, the need for high qualified manpower and long lag time between sample collection and decisions. This final response time may typically vary between 45 minutes and 2 hours, depending on the type of analysis required by the olive oil carried by the delivery vehicle. Therefore, within a context in which it is imperative to guarantee food safety and quality, the availability and the need for confirmatory protocols based on faster, simpler to use and more reliable detecting tools is critical. The implementation of alternative approaches to these classical methodologies thus represents a necessary step towards more efficient and modern monitoring, management and control systems. ${ }^{4}$

Over the last few years, near infrared spectroscopy (NIRS) has been one of the most important techniques to be used in the food and feed laboratories. ${ }^{5}$ This technology shows great potential and already plays an important role due to its ability for rapid, low-cost, non-contaminant and non-destructive qualitative and quantitative analysis of a high variety of products and constituents. These features are of particular interest to achieve costeffective and fit-for-purpose methodologies requiring less technical training and manpower. In addition to the time- and cost-saving benefits, recent advances in NIRS instrumentation have allowed the possibility of developing tools that enable in situ collection of spectral information. ${ }^{6,7}$ Consequently, a new paradigm concerning sampling and analysis of bulk raw materials could emerge, in which both tasks could be performed at the same time leading to a real-time decision-making process.

The olive oil sector is increasingly interested in the application of NIRS, ${ }^{8}$ which has proved to be one of the most efficient and advanced tools for the determination and prediction at laboratory level of the major parameters on olive oils. Despite this progress, little research has been done into the in situ analysis of large volumes of olive oils in bulk using near infrared spectroscopy (NIRS). ${ }^{9,10}$ For this reason, work has been carried out within the framework of a research project at the Department of Agrolivestock Systems Engineering (University of Córdoba, Spain) to design and build a fiber-optic probe. This tool, interfaced to a NIRS instrument, could serve to sample and analyse olive oils directly in the tanker trucks.

The aim of this work is to perform a preliminary evaluation of the designed fiber-optic probe for in situ quality analysis of olive oils at the reception level of packing plants. The specific objectives of the evaluation were to optimize the collection of NIRS spectra with the probe coupled to a Fourier transform (FT)-NIR instrument, as well as to make a spectral comparison with a laboratory probe with which prior work had been done.

\section{Materials and methods Instrumentation}

A Matrix-F FT-NIR instrument (Bruker Optics, Germany) was used to perform transmittance analysis of olive oils 


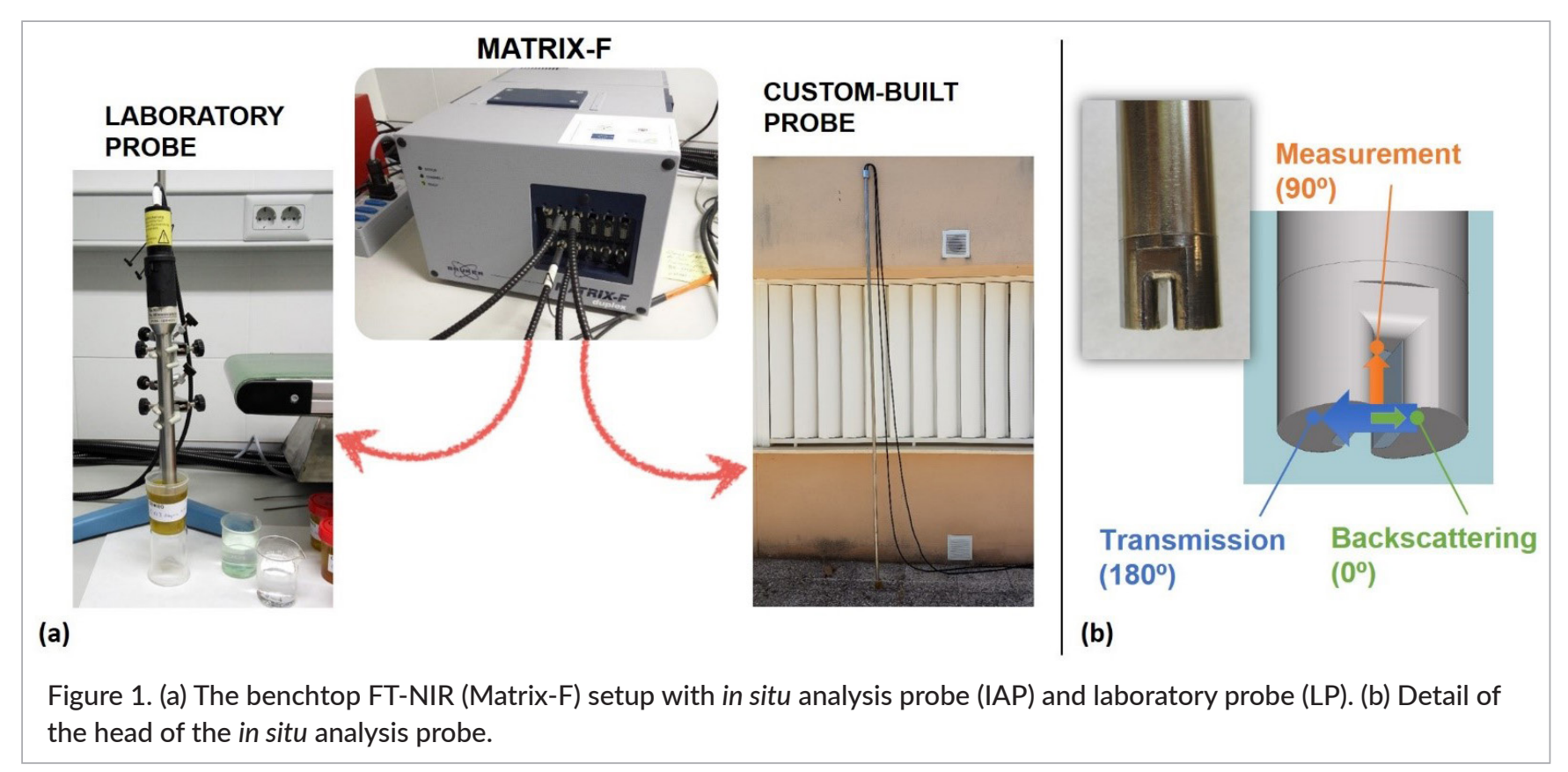

samples (Figure 1a). This equipment supports multiple modes of analysis and features six channels to connect fiber optic sensors.

Two different types of fiber-optic probes were used in this work (Figure 1a). First, the probe for in situ analysis of liquids (henceforth referred to as IAP) is a prototype which is characterized by a stainless-steel body of $3 \mathrm{~m}$ in length and $25 \mathrm{~mm}$ in diameter. Two fiber optic cables, whose length is $50 \mathrm{~m}$, were used to interface this probe to the FT-NIR instrument, one for collection and one for illumination. The irradiation bundle consists of a set of 19 fibers, while the measurement bundle contains 7 fibers (both bundles comprise fibers of $220 \mu \mathrm{m}$ in diameter). The head of the probe has a path length of $4 \mathrm{~mm}$. It was designed to increase the speed of the liquid at the measurement zone and includes three measuring channels $\left(90^{\circ}\right.$, transmission or $180^{\circ}$ and backscattering or $0^{\circ}$ ) (Figure $1 \mathrm{~b}$ ), the use of which depends on the sort of liquid to be analysed. Transmission mode was implemented in this work.

On the other hand, a laboratory probe (LP) was also used connected to the Matrix-F (Figure 1a). This probe served as a benchmark, since a previous work with it resulted in the development of equations for prediction of a range of key parameters regarding quality determination of different kinds of olive oils. In this case, the fiber optic cables are $2 \mathrm{~m}$ in length and the path length is $2 \mathrm{~mm}$. Transmittance was also the mode of analysis with this probe.

\section{Spectral acquisition optimization and repeatability study}

A set of two samples (set 1), one of extra virgin olive oil and one of lampante olive oil, was used to carry out the optimization of the spectral analysis with IAP. These samples, containing 5 litres per sample, were directly taken from the tanks of the collaborating industry. Once the samples were received at the laboratory, one litre of each sample was poured into two different glass containers for analysis.

The purpose of this part of the study was to test and fine-tune the configuration and operation of the probe. A set of tests was designed to assess strategies and procedures of making spectral measurements, as well as to address the evaluation of the stability of the IAP over time. To this end, a 5-week study was carried out in which three tests per day were performed, a total of 7 days within that period. Each test was based upon the collection of spectra following the sequence: (1) Recording of reference spectra. (2) A set of 20 spectra of lampante olive oil. (3) Cleaning. (4) A set of 20 spectra of extra virgin olive oil. (5) Cleaning. OPUS v.7.0 (Bruker Optics) software was used for spectral acquisition.

The noise level along the spectral range available from the spectrometer $(834.2-2502.4 \mathrm{~nm})$ was evaluated after the full set of tests was performed. A first derivative pre-treatment with a single-unit gap and five data-point smoothing was applied to the spectral 
data for this purpose. Furthermore, spectral repeatability was also assessed by using the Root Mean Square (RMS) statistic, calculated as the averaged root mean square of differences between replicates of the spectral values.

\section{Spectral comparison}

A different sample set (set 2) was used for the comparative study between IAP and LP. For the purpose of simulating the range of types of olive oils in a packing plant, the new set ( $N=42$ samples) selected was directly obtained from the tanks of the industry and was characterized by a high heterogeneity, containing both filtered and unfiltered olive oils which consisted of mixtures from a variety of sources and different organoleptic and physico-chemical properties. Moreover, lampante olive oils with a high level of impurities were also included. Reference analysis for the most important quality parameters in olive oils (acidity, peroxide value, methyl esters, ethyl esters, K232, K270, fat content, moisture etc.) was available for all samples.

In order to compare the spectral response between both probes, a standardization protocol was initiated following the Shenk procedure. ${ }^{11,12}$ NIR analysis was carried out in duplicate for each sample by both probes under controlled environmental conditions. IAP was considered as satellite during the cloning process, whereas LP as master. Two strategies using different sample groups as "cloning set" were tested to generate standardization matrices: (1) sample closest to the center of the population (STD1); and (2) 10 samples were selected with a view to covering the whole range of the spectral variability (STD2). The "validation set" comprised 32 samples and was the same in both cases. The CENTER algorithm included in the WinISI II version 1.50 software package (Infrasoft International, Port Matilda, PA) served to treat the spectral database so as to rank spectra according to their Mahalanobis distance ( $\mathrm{H}$ distance). Results of standardization were evaluated using the RMS(c) statistic, for all 32 samples selected for the validation set. The RMS(c) statistic was used to compare spectra for the same sample obtained with both probes, and was calculated using the CONTRAST algorithm included in the WINISI software:

$$
\operatorname{RMS}(c)=10^{6} \sqrt{\frac{\sum_{i=1}^{n} D_{i}^{2}-\frac{\sum_{i=1}^{n} D_{i}^{2}}{n}}{n-1}} ; D_{i}=y_{i m a s t e r}-y_{\text {isatellite }}
$$

where $y_{i \text { master }}$ and $y_{i \text { satellite }}$ are the $\log (1 / R)$ values of two spectra of a single sample scanned in master and satellite at a given wavelength.

\section{Results and discussion Determination of the useful wavelength range and repeatability study interpretation}

The assessment of noisy regions is a crucial target so as to determine the total useful wavelength range of the tool being evaluated. It has been examined that the light power propagating in a fiber decays exponentially with length due to absorption and scattering losses. Thus, there exists a direct relationship between the length of the fiber-optic cables along with the properties of the samples analyzed and the level of noise. As stated before, in this study, the length of the fibers to connect the IAP and the Matrix- $F$ was $50 \mathrm{~m}$, which a priori does not mean that performance would have to be significantly affected. Previous work developed by our research group demonstrated that the Matrix-F reaches lengths of up to $100 \mathrm{~m}$ without compromising spectral repeatability. ${ }^{13}$ Resulting spectra using IAP for extra virgin olive oil from sample set 1 are shown in Figure 2a.

It can be noted that regions of noise appear at the beginning and at the end of the spectral region, which is not a rare behaviour when dealing with fiber-optic probes. Figure $2 b$ shows extra virgin olive oil spectra transformed using a first-order derivative, a single-unit gap and five data-point smoothing. This type of derivative degrades the signal/noise ratio, so that its application leads to the presence in some spectral regions of random oscillations in absorbance values (noise). The most relevant absorption bands observed in the spectra were located at $1208 \mathrm{~nm}, 1414 \mathrm{~nm}, 1725 \mathrm{~nm}, 1760 \mathrm{~nm}$ and $2144 \mathrm{~nm}$, which are typically linked to this type of olive oil. Nevertheless, there are some other distinctive absorption peaks in this kind of products, such as at $2308 \mathrm{~nm}, 2350 \mathrm{~nm}$ or $2380 \mathrm{~nm}$, which are not present due to the presence of noise in that region. After the evaluation of the noisy regions, the optimum working range was selected as 1150-2149 nm (Figure 2b).

Spectral repeatability was assessed using the RMS statistic. Table 1 summarizes the main results of the evaluation, showing MEAN and STD values of this statistic both for lampante olive oil and extra virgin olive oil (sample set 1). NIRS analysis using IAP resulted in a lower 


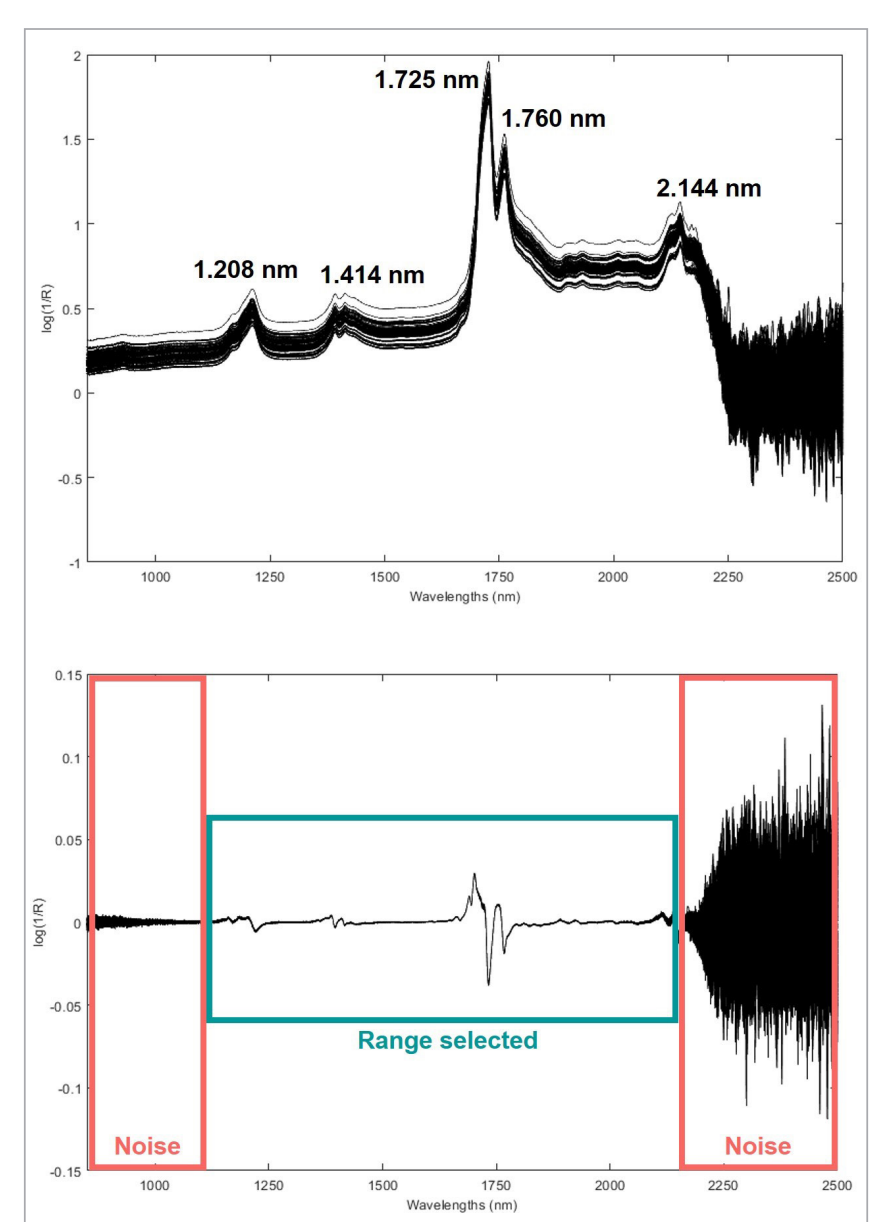

Figure 2. Spectra of extra virgin olive oil obtained with the prototype (a) raw spectra and (b) first derivative spectra.

Table 1. Values of MEAN and STD of the RMS for the sample set $1(n=2)$. Units are in $\mu \log (1 / R)$.

\begin{tabular}{|l|c|c|}
\hline & Mean (RMS) & STD (RMS) \\
\hline Lampante olive oil & 12,630 & 12,959 \\
\hline Extra virgin olive oil & 2998 & 3263 \\
\hline
\end{tabular}

spectral repeatability for lampante olive oil than for extra virgin olive oil. The MEAN and STD values of this work for extra virgin olive oil are in line with those reported in other studies. ${ }^{14}$ However, for lampante olive oil are generally higher in this case. This could be attributable to the fact that filtered olive oils samples may represent a better alternative for NIRS analysis by IAP than unfiltered olive oils, which was the case of the lampante olive oil of sample set 1 . Furthermore, this sample especially contained many suspended particles that may have contributed to hinder the achievement of a better spectral repeatability.
Table 2. RMS(c) statistic for the validation set $(n=32)$ in master and satellite before and after cloning. Units are in $\mu \log (1 / R)$.

\begin{tabular}{|l|l|}
\hline \multicolumn{1}{|l|}{} & RMS(c) \\
\hline Mas. vs Mas. & 4052 \\
\hline Mas. vs Sat. & 131,805 \\
\hline Mas. vs Sat.STD1 & 19,748 \\
\hline Mas. vs Sat.STD2 & 30,836 \\
\hline
\end{tabular}

\section{Comparing the spectral response}

Sample set 2 served to contrast IAP with LP. Results for the evaluation of standardization matrices are shown in Table 2, which lists RMS(c) values for each matrix (STD1 and STD2). Significant differences can be observed between master (LP) and satellite (IAP) prior to standardization $[131,805 \mu \log (1 / R)]$. The best spectral fit was obtained when applying the STD1, which yielded a fit of 19,748 $\mu \log (1 / \mathrm{R})$.

Figure 3 shows the spectral adjustment achieved after applying the STD1 matrix to the average spectrum of the validation set. Despite RMS(c) remains considerably higher in value than for the master (Table 2), STD1 manages to reduce significantly the initial spectral differences between master and satellite (Figure 3). However, standardization does not completely succeed in matching the spectral response of both probes. This could be expected taking into account the important differences between IAP and LP before the cloning, as well as the fact that IAP is still a prototype, with which more work needs to be done to achieve its optimal performance. Moreover, it is worth noting that the designs of IAP and LP are significantly different, for instance, concerning the length of their fiber optic cables $(50 \mathrm{~m}$ versus $2 \mathrm{~m}$, respectively), and whose influence on the results should be considered.

\section{Conclusions}

This work represents a first step towards the implementation of a methodology for in situ quality analysis of olive oils directly at the reception of packing plants. The evaluation of raw materials before entering the production chain is of high value in order to guarantee food safety and quality but is not without challenges. Application of innovative technologies such as near infrared spectroscopy in this context could mean the possibility of devel- 


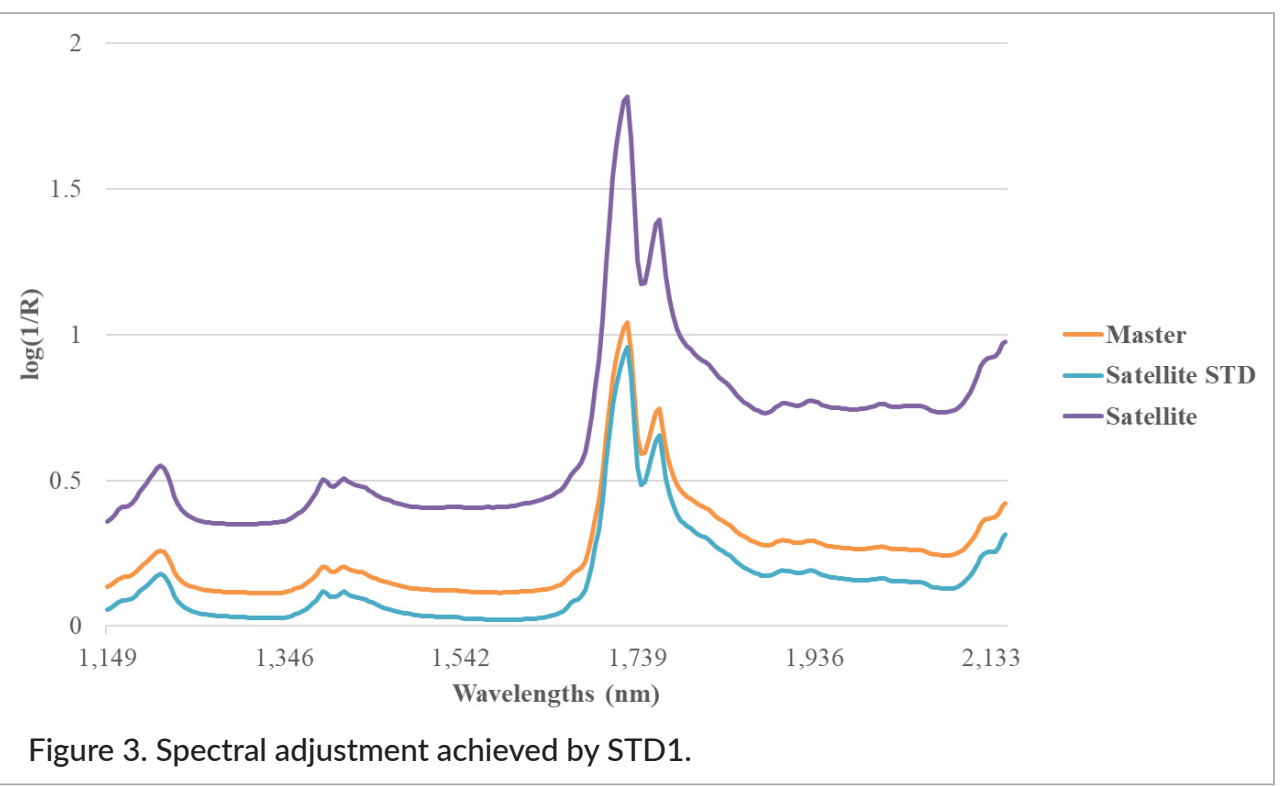

oping faster and more cost-effective protocols. The spectral response of the fiber-optic probe especially designed for this purpose has been tested. Results show that its spectral repeatability can be considered as acceptable particularly when analyzing filtered olive oils, whereas unfiltered olive oils such as olive oils of lampante category will need to be evaluated more in-depth to determine the real applicability in this case. Furthermore, results of the comparative study between the in situ analysis probe and the laboratory probe indicate that there were important spectral differences, which is reasonable given the highly different designs of both probes. Despite this, the standardization process managed to significantly reduce the initial differences between master and satellite. Nonetheless, a more thorough study will need to be conducted in order to test the new probe in a variety of conditions, as well as to develop calibration models based on a larger spectral library of samples and evaluate its potential and feasibility for application in real situations.

\section{References}

1. European Commission, "Regulation (EC) No. 882/2004 of the European Parliament and of the Council of 29 April 2004 on Official Controls Performed to Ensure the Verification of Compliance with Feed and Food Law, Animal Health and Animal
Welfare Rules", Off. J. Eur. Union L 165(April), 1-141 (2004).

2. International Olive Council, Quality Management for the Olive Oil Industry: Packing Plants. T.33-2/Doc. no. 4 (2006).

3. International Organization for Standardization (ISO), ISO 5555:2001 - Animal and vegetable fats and oils -Sampling (2001).

4. F. Cheli, A. Campagnoli, L. Pinotti and V.D. Orto, "Rapid methods as analytical tools for food and feed contaminant evaluation: methodological implications for mycotoxin analysis in cereals", in Food Production: Approaches, Challenges, and Tasks. Intech Open (2012). http://www.intechopen.com/books/ food-production-approaches-challenges-and-tasks [accessed Oct 16 2017]

5. E. Fernández-Ahumada, A. Garrido-Varo,

J.E. Guerrero-Ginel, D. Pérez-Marín and T. Fearn, "Taking NIR calibrations of feed compounds from the laboratory to the process: Calibration transfer between predispersive and postdispersive instruments", J. Agric. Food Chem. 56, 10135-10141 (2008). https://doi.org/10.1021/jf801881n

6. E. Zamora-Rojas, D. Pérez-Marín, E. de Pedro-Sanz, J.E. Guerrero-Ginel and A. Garrido-Varo, "Handheld NIRS analysis for routine meat quality control: database transfer from at-line instruments". Chemometr. Intell. Lab. Syst. 114, 30-35 (2012). https://doi. org/10.1016/j.chemolab.2012.02.001 
7. A. Garrido-Varo, S. Vega, F. Maroto-Molina, M. José de La Haba and D. Pérez-Marín, "On-site quality control of processed land animal proteins using a portable micro-electro-mechanical-systems near infrared spectrometer", J. Near Infrared Spectrosc. 24, 47 (2016). https://doi.org/10.1255/jnirs.1192

8. S. Armenta, J. Moros, S. Garrigues and M. De La Guardia, "The use of near-infrared spectrometry in the olive oil industry", Crit. Rev. Food Sci. Nutrit. 50, 567-582 (2010). https://doi. org/10.1080/10408390802606790

9. A. Garrido-Varo, M.-T. Sánchez, M.-J. De la Haba, I. Torres and D. Pérez-Marín, "Fast, low-cost and non-destructive physico-chemical analysis of virgin olive oils using near-infrared reflectance spectroscopy", Sensors 17, 2642 (2017). https://doi. org/10.3390/s17112642

10. N. Nenadis and M.Z. Tsimidou. "Perspective of vibrational spectroscopy analytical methods in on-field/official control of olives and virgin olive oil", Eur. J. Lipid Sci. Technol. 119, 1-18 (2017). https://doi. org/10.1002/ejlt.201600148

11.J.S. Shenk and M.O. Westerhaus, "New standardization and calibration procedure for NIRS analytical systems", Crop Sci. 1694-1696 (1991). https://doi. org/10.2135/cropsci1991.0011183X0031000600 $64 x$

12.J.S. Shenk, M.O. Westerhaus and S.M. Abrams, "Protocol for NIR calibrations: monitoring analysis results and recalibration. Near Infrared Spectroscopy (NIRS): analysis of forage quality", in Agriculture Handbook No. 643, Ed by G.C. Marten and F.E. Barton. USDA, ARS, pp. 104-110 (1989).

13. A. Sanchez-Bonilla, A. Garrido-Varo, F. MarotoMolina, J.E. Guerrero- Ginel and B. de La RozaDelgado, "Influence of the fiber optic length on the repeatability of the near infrared spectroscopy signal", in Proceedings of the 17th International Conference on Near Infrared Spectroscopy. Foz do Iguassu, Brazil (2015).

14.J.M. Chinchilla-Hornos, A Laboratory Liquid Probe Interfaced to a FT-NIRS Instrument for the Prediction of Physico-Chemical Parameters as Quality Indicators in Virgin Olive Oil. Master thesis, University of Córdoba (2014). 